\title{
Cornell Caltech Atacama Telescope primary mirror surface sensing and controllability
}

Daniel MacDonald, David Woody, C. Matt Bradford, Richard Chamberlin, Mark Dragovan, et al.

Daniel MacDonald, David Woody, C. Matt Bradford, Richard Chamberlin, Mark Dragovan, Paul Goldsmith, Simon Radford, Thomas Sebring, Jonas Zmuidzinas, "Cornell Caltech Atacama Telescope primary mirror surface sensing and controllability," Proc. SPIE 7012, Ground-based and Airborne Telescopes II, 701211 (17 July 2008); doi: 10.1117/12.790528

Event: SPIE Astronomical Telescopes + Instrumentation, 2008, Marseille, France 


\title{
Cornell Caltech Atacama Telescope Primary Mirror Surface Sensing and Controllability
}

\author{
Daniel MacDonald ${ }^{\mathrm{a}}$, David Woody ${ }^{\mathrm{b}}$, C. Matt Bradford ${ }^{\mathrm{a}}$, Richard Chamberlin ${ }^{\mathrm{b}}$, Mark Dragovan ${ }^{\mathrm{a}}$, Paul \\ Goldsmith $^{\mathrm{a}}$, Simon Radford ${ }^{\mathrm{b}}$, Thomas Sebring ${ }^{\mathrm{c}}$, Jonas Zmuidzinas ${ }^{\mathrm{a}}$, \\ a Jet Propulsion Lab, California Institute of Technology, Pasadena, CA, USA 91109 \\ ${ }^{b}$ California Institute of Technology, Pasadena, CA, USA 91125 \\ ${ }^{\mathrm{c} C}$ Cornell University, Ithaca, NY, USA 14853
}

\begin{abstract}
To meet the $10 \mu \mathrm{m}$ RMS half wavefront error requirement for the $25 \mathrm{~m}$ diameter Cornell Caltech Atacama Telescope (CCAT), active control of the approximately 200 primary mirror panels is required. The CCAT baseline design includes carbon fiber aluminum honeycomb sandwich mirror panels. Distortions of the panels due to thermal gradients, gravity and the mounting scheme need to be taken into consideration in the control system design. We have modeled the primary mirror surface as both flat and curved surfaces and have investigated mirror controllability with a variety of sensor types and positions.

To study different mirror segmentation schemes and find acceptable sensor configurations, we have created a software package that supports multiple segment shapes and reconfigurable panel sizing and orientation. It includes extensible sensor types and flexible positioning. Inclusion of panel and truss deformations allows modeling the effects of thermal and gravity distortions on mirror controllability.

Flat mirrors and curved mirrors with the correct prescription give similar results for controlled modes, but show significant differences in the unsensed flat mirror modes. Both flat and curved mirror models show that sensing schemes that work well with rigid, thermally stable panels will not control a mirror with deformable panels. Sensors external to the mirror surface such as absolute distance measurement systems or Shack-Hartmann type sensors are required to deal with panel deformations. Using a combination of segment based sensors and external sensors we have created a promising prototype control system for the CCAT telescope.
\end{abstract}

Keywords: CCAT, large telescopes, segmented mirrors, mirror alignment, telescope control

\section{INTRODUCTION}

A primary mirror for a large telescope requires a very precise surface, leading to designs in which the primary mirror is composed of a number of smaller, easier to manufacture mirrors or panels. Changing environmental conditions for a telescope such as temperature, wind speed and zenith angle of the mirror alter the alignment of the panels and compromise the optical surface, so panels are actuated to allow them to be repositioned in real time.

The purpose of a surface control system is to maintain the surface figure using a set of sensors to measure the surface distortions and a set of actuators to correct the distortions. The assumption in this approach is that the surface has been set to the desired shape using an astronomical wave front measuring system and the sensor readings are known for this ideal shape.

Ground-based and Airborne Telescopes II, edited by Larry M. Stepp, Roberto Gilmozzi, Proc. of SPIE Vol. 7012, 701211, (2008) · 0277-786X/08/\$18 - doi: 10.1117/12.790528 
An idealized segmented telescope with an active surface is shown in Figure 1. The support structure or truss is shown in dark gray. The truss is connected to the telescope mount through the drive system and is the structure from which the panels are supported. The actuators float the panels on the truss and allow the panel positions to be individually adjusted. Since all the actuator in one panel move in the same direction, flexures connect the actuators to the panel and isolate the panels from stresses created by changing actuator head geometry. The panels, which can be monolithic or contain support structures such as warping harnesses or Wiffle trees ${ }^{[1]}$, maintain the integrity of the optical surface they support. The panels are the smallest unit of active control in the system, but may contain a number of individual unactuated mirrors or tiles.

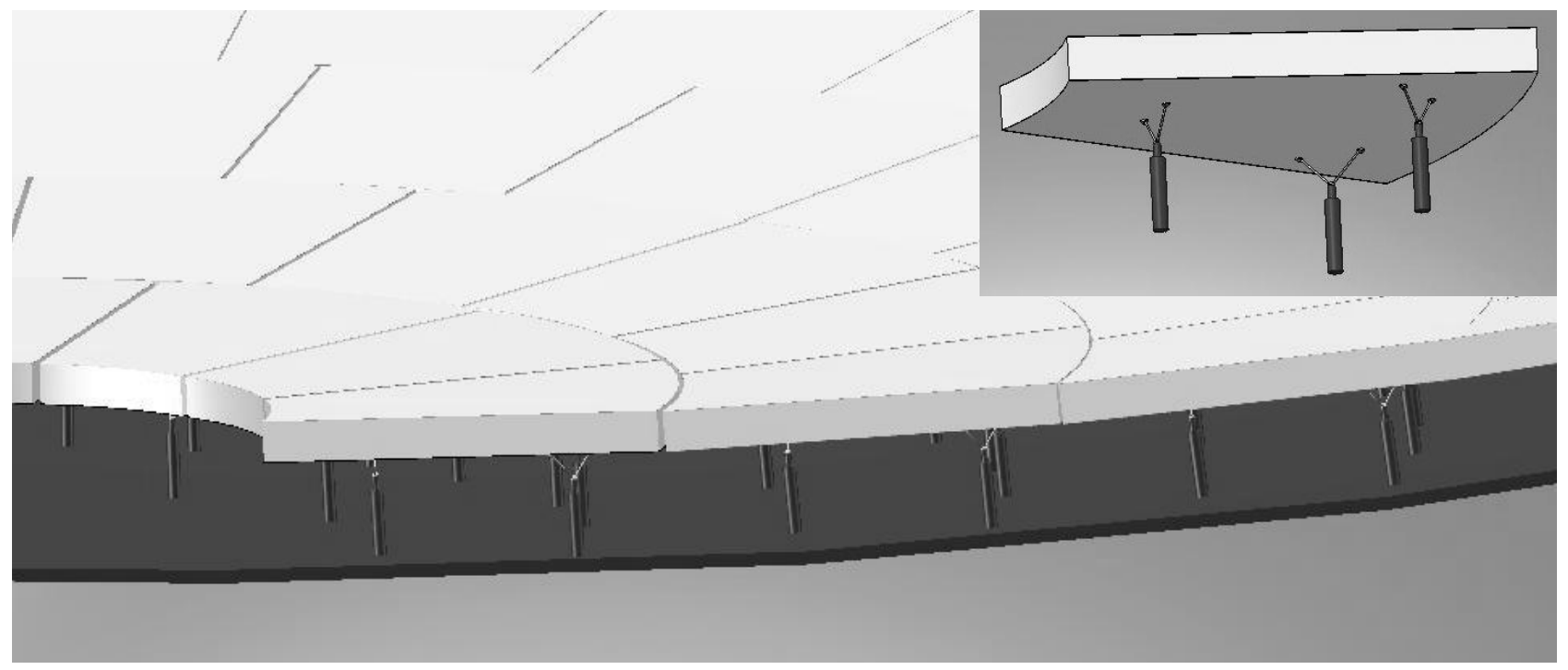

Fig. 1. Segmented telescope cross section with single panel inset

\section{SENSORS}

We examined two basic types of sensors for mirror control. Panel based sensors measure panel to panel motions without reference outside of the mirror. External sensors compare mirror positions to an external reference frame. The most basic panel sensor measures the displacement of a segment surface relative to an adjacent segment. Figure 2 shows a prototype displacement sensor, the Keck edge sensor. To maintain the mirror surface, the front surface of the panels and their normals must be continuous across the gaps between panels. Pure displacement sensors, when placed at the edge of the panels are not sensitive to the surface normal differences between panels called the dihedral angle, but placed offset from the edge like the $55 \mathrm{~mm}$ offset shown in the schematic in Figure 2, pairs of sensors have an effective dihedral angle sensitivity which allows for controls system made entirely from offset displacement sensors. A number of different technologies such as capacitive, inductive and optical sensors can be used to create edge sensor systems. Some edge sensor configurations have a dependency on the gap between the segments, but we consider only gap insensitive edge sensors in this study. Dedicated dihedral angle sensors which measure only the difference between the normals to the panels at the edges can also be used instead of or in addition to offsetting of displacement sensors. External sensors measure to a reference frame outside of the primary mirror such as the secondary mirror. We consider absolute distance measuring sensors such as interferometric laser metrology sensors and absolute angle measuring sensors such as Shack-Hartmann systems as our external sensors. To be effective, the external sensors reference frame must be known relative to the mirror. They therefore require an additional sensor system to link reference frame motions back to the mirror coordinate system. 

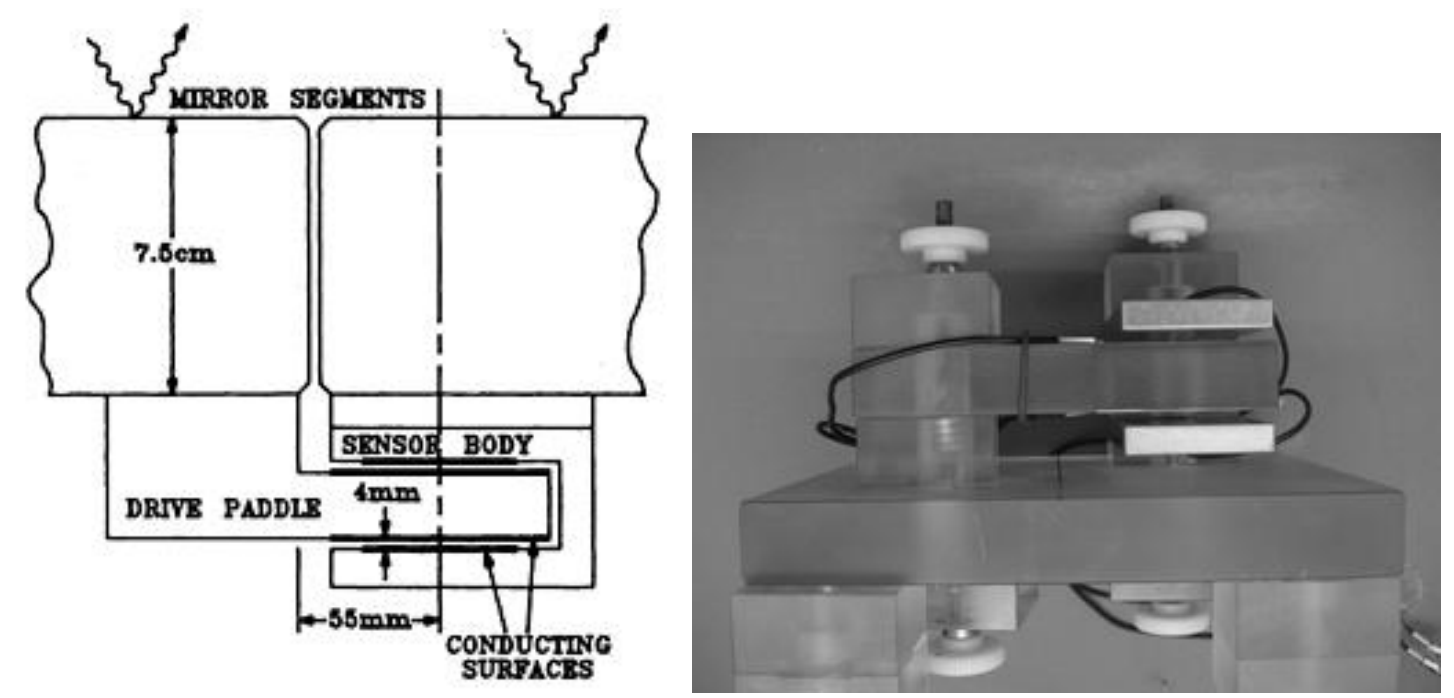

Fig. 2. Keck edge sensor. Left-schematic Right-Prototype

\section{CONTROL MATRIX}

To control the mirror, the control system requires a mapping of sensor readings into the actuator motions required to correct the mirror position. This mapping, the control matrix, encapsulates the geometric relationship between sensor measurements and actuator drive vectors. Determining the control matrix for a certain configuration of panel, sensor and actuator geometry is the heart of the control system. The first step in creating the control matrix is to create its inverse called the response matrix. The response matrix is the set of sensor outputs for a certain mirror geometry, which is indexed by the panel motions used to create it. The matrix is then inverted and can be analyzed to determine its control properties.

The process of creating the control matrix is illustrated with a a simple example, a 4 panel mirror shown in Figure 3. The figure shows the example mirror with actuator positions shown in red and edge and dihedral angle sensors shown in blue and yellow respectively.
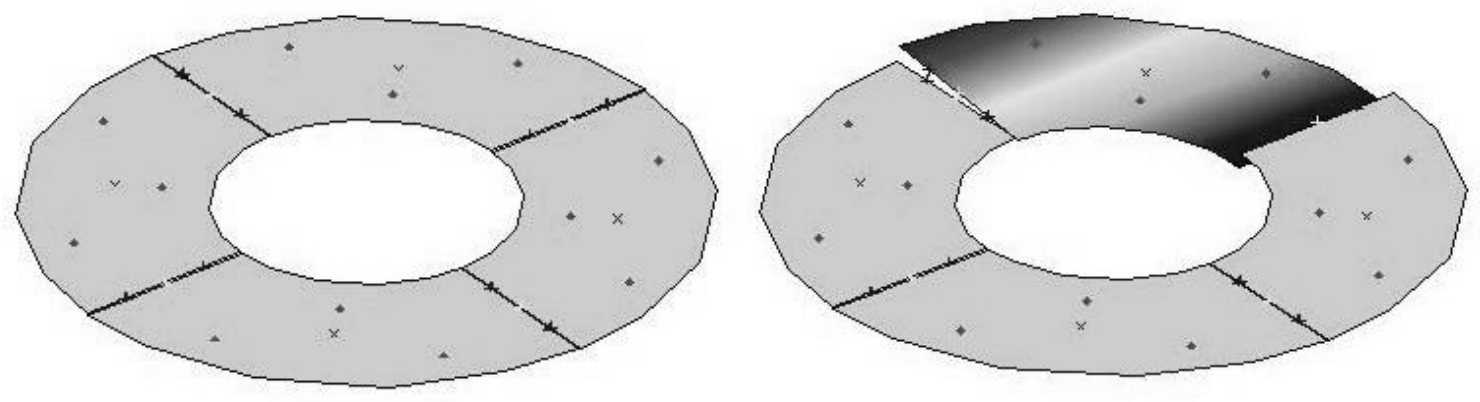

Fig. 3. Four panel example mirror with actuator locations shown as diamonds, sensors locations as $+s$, and panel centers as Xs. Left - initial mirror position. Right - mirror with one panel tipped.

The response matrix is created one row at a time by exercising one of the degrees of freedom on one of the panels in a mirror and recording the sensor readings for all of the sensors in the system as columns. The right panel of Figure 3 shows the mirror with one degree of freedom exercised, The example system has 12 sensors, two edge sensors and an angle sensor on each edge. By actuating all three DOF for each of the 4 panels, the $12 \times 12$ response matrix is created. 
If all the possible degrees of freedom of the mirror are actuated, a response matrix will be created that spans all the possible mirror configurations.

The response matrix maps segment motions to sensor readings. Inverting this matrix will map sensor readings to segment motions giving the control matrix. The response matrix in general is not square, its dimensions are the degrees of freedom in the mirror by the number of sensors. To have the possibility of completely controlling the mirror, the system requires at least as many sensors as degrees of freedom in mirror motion. For practical systems the number of sensors is greater than the mirror DOF. We create a psuedoinverse of the response matrix using a Singular Value Decomposition (SVD). Along with providing an inverse, the SVD also gives the eigenmodes of the sensor-actuator system. This mapping to an eigenmode basis for the system enables the control system to treat the different mirror modes individually and can be used to determine the controllability of the mirror sensing configuration.

The eigenvalues of the control matrix correspond to how well determined a particular eigenmode is based on the sensor readings. Small eigenvalues are characteristic if badly determined - badly controlled - modes while large eigenvalues are characteristic of well defined modes. Modes with a zero eigenvalue are completely unsensed. The eigenmodes give the shape of the normal modes of the mirror. These modes are not physical modes of the system such as vibrational modes. Rather, they represent the normal modes of the mirror in response to sensor measurements.

A more directly interpretable way of representing the mirror controllability than the eigenvalue is the Error Multiplier (EM) which measures the actuator motions resulting from a gaussian noise distribution in sensor outputs. For any mode of the mirror, the EM is the RMS actuator motion divided by the RMS sensor noise which is responsible for the motion. Thus multiplying sensor noise by the EM gives the average amount the actuators are moved which is the distortion introduced into the surface as a result of the noise in the sensor readings. The EM then is a measure of the controllability of that individual mode and since mirror modes are orthogonal, the EMs can be added in quadrature give the controllability of the entire system. The 12 normal modes of the 4 segment example mirror are shown in Figure 4.

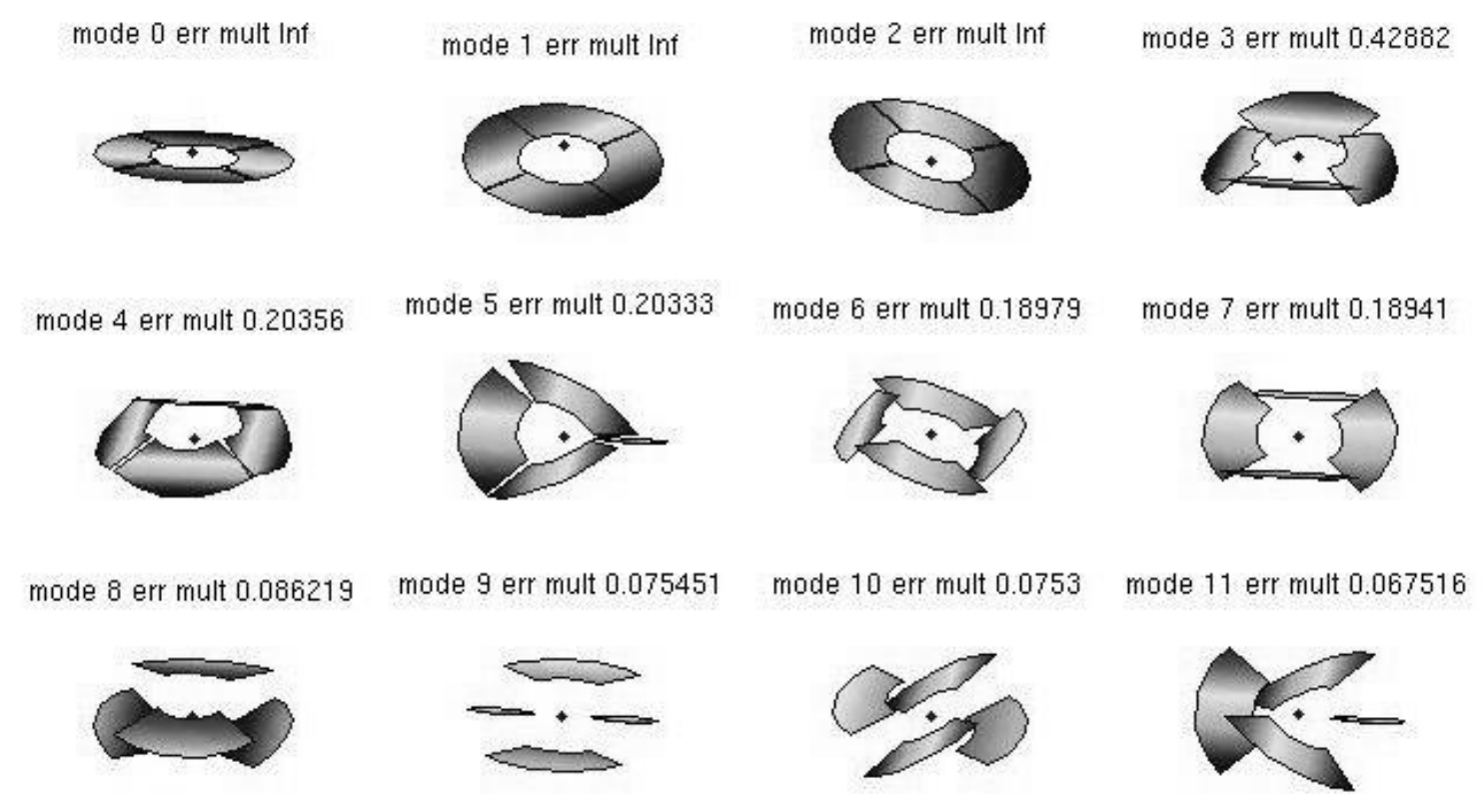

Fig. 4. Mirror modes for example mirror in order of decreasing Error Multiplier.

The modes are ordered in decreasing EM value which corresponds to increasing eigenvalue. This puts the least well determined modes at the upper left of the figure and the best determined modes at the lower right. This can be seen in 
the figure as how much more distance there is between the sensor endpoints in mode 11, the best determined mode, as compared to mode 3 . The total error multiplier excluding the first three modes is 0.6 .

The first three modes of the system shown in Figure 4 with infinite error multipliers are the unsensed solid body motions of the entire mirror: global tip, tilt and piston. The example mirror is entirely sensed by panel based sensors so it has no sensitivity to motions of the entire mirror relative to external reference frames such as the model fixed reference frame.

\section{MIRROR SEGMENTATION}

CCAT is a $25 \mathrm{~m}$ aperture submillimeter telescope with a goal of operating at a wavelength of $200 \mu \mathrm{m}$. This leads to a $9.5 \mu \mathrm{m}$ total half wavefront requirement for the system of which the primary mirror system is allocated $7 \mu \mathrm{m}$ including panel manufacturing errors. The early baseline segmentation layout for the CCAT primary mirror is 7 rings of $1.6 \mathrm{~m}$ panels. We consider two layouts for the primary, a keystone layout which has identical panels in each ring and a hexagonal layout where each 60 degree section of the mirror has unique panels. The inner three rings of the keystone layout is shown in the left panel of figure 5 and the inner 3 rings of the hexagonal layout shown in the right panel of Figure 5. The locations of $55 \mathrm{~mm}$ offset edge sensors are shown.
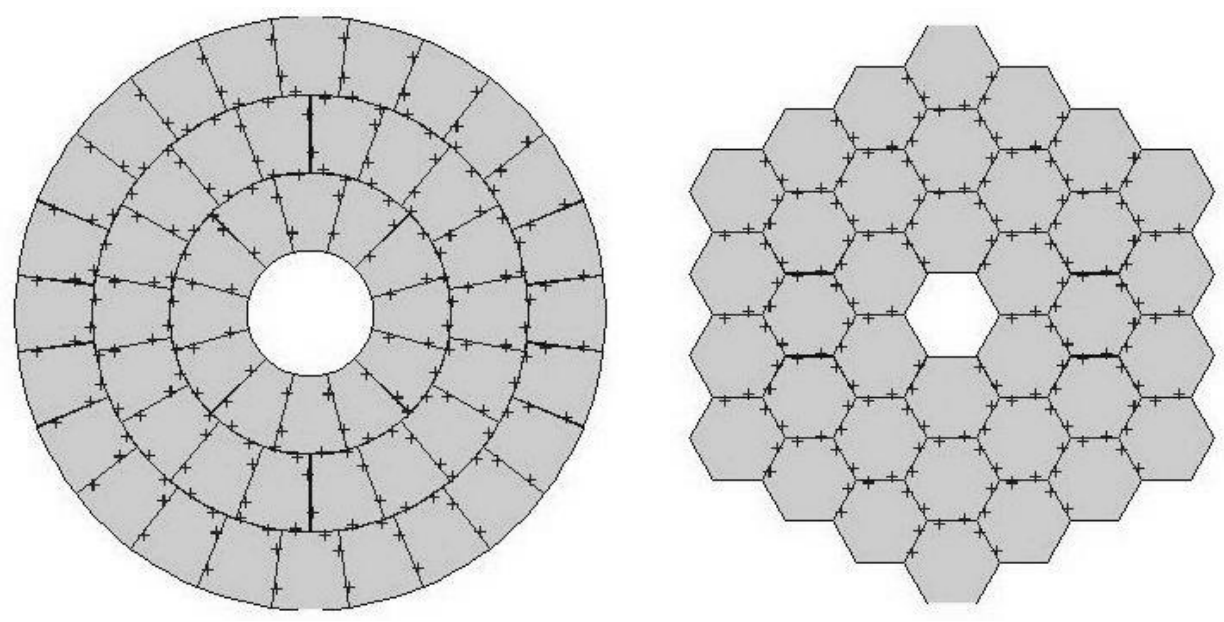

Fig. 5. 3 ring segmented mirrors with 55mm offset edge sensor locations. Left-keystone Right-hexagon

\section{HEXAGONAL VS. KEYSTONE MODELS}

To investigate the differences between hexagonal and keystone segmentation geometries, we used the same sensor configuration for both geometries and varied the number of rings of panels in the mirror. We used a Keck like sensor configuration for this test with two edge sensors per side offset by $55 \mathrm{~mm}^{[2]}$. For differing numbers of rings of panels the modes of the two geometries were very consistent. Both the mode order and shape were very similar for the $1.6 \mathrm{~m}$ panel, 2 to 7 ring mirror configurations that were studied. The first three sensed modes and their error multipliers for each geometry with 7 rings are shown in Figure 6. For the keystone mirrors the most difficult mode to control is astigmatism and for hexagonal systems it is focus.

The total error multipliers in Table 1 indicate a constant 3 times better controllability for hexagonal systems over the range of mirror sizes. The total error multiplier is increasing linearly with the number of rings showing that mirrors with more rings are moderately more difficult to control than are smaller systems. 

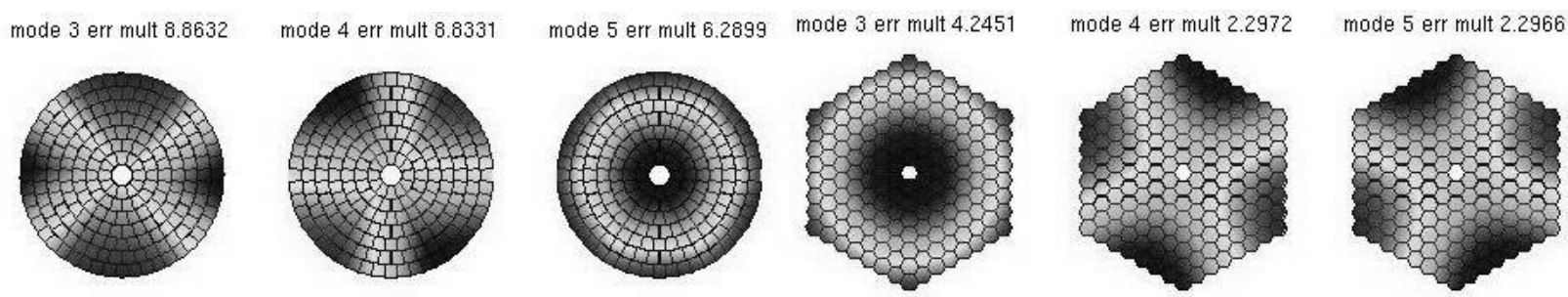

Fig. 6. Least controlled modes. Left-Keystone Right-Hexagon

Table 1. Total error multipliers for study mirror configurations

\begin{tabular}{|lrrrrrr|}
\hline \multicolumn{7}{c|}{ Mirror Total Error Multipliers } \\
\# of rings & 2 & 3 & 4 & 5 & 6 & 7 \\
Keystone & 9.9 & 10.3 & 11.8 & 13.1 & 14.4 & 15.6 \\
Hexagonal & 3.5 & 3.8 & 4.3 & 4.8 & 5.5 & 6.1 \\
\hline
\end{tabular}

\section{FLAT VS. CURVED MODELS}

Control systems for large telescopes have, up till now, been designed using flat mirror models. Since the control matrix encapsulates the mirror geometry for the control system, the geometric relation between the sensors and the actuators for a flat mirror has been built into the control system. As telescopes become larger and their optics faster (more highly curved), this is becoming an increasingly poor assumption. The most obvious change is the relationship between the actuator motions of the different panels. For a flat mirror such as the one in the left panel of Figure 7, driving all the actuators the same amount results in the entire mirror being pistoned. This does not change any readings of panel based sensors and is reflected in an unsensed piston mode as seen in Figure 4. For a spherical mirror driving all the actuators the same amount towards the center of the sphere results in a change in the size of the mirror, but the relationship between the panels is unchanged and the mode is unsensed For any other curved mirror such as the parabolic mirror in the right panel of figure 7, each segments actuators drive in a unique direction toward differing focal points. Driving all the actuators the same amount then results in a change in the relationship between all the panels and can be seen as a sensed mirror mode.
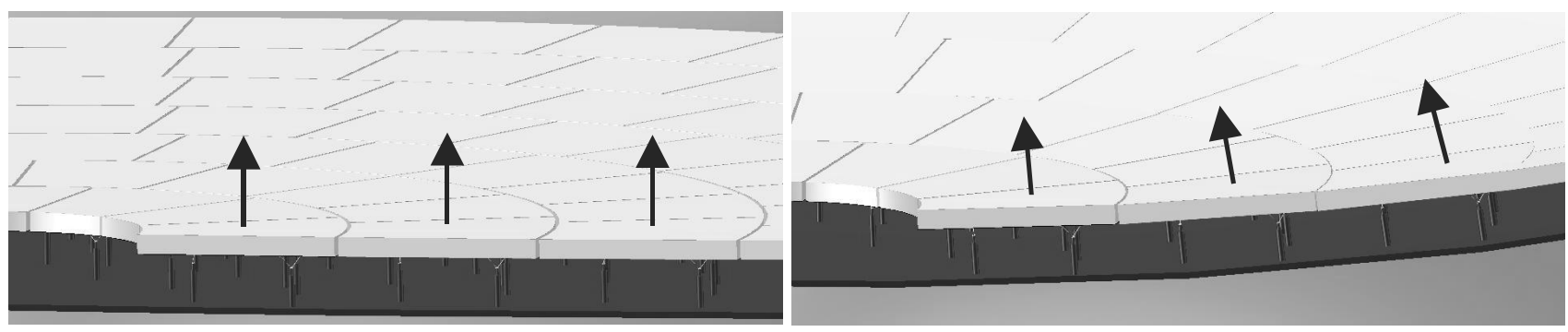

Fig. 7. Left - Flat mirror actuation all in one direction. Right - For curved mirror each panel's actuation has a unique direction

Since the panels all move in different directions, curved mirrors can not be driven in purely piston mode, it is no longer a mode of the system. The tip and tilt like modes can now be sensed since they result in a change in relationship between the panels. These modes are not well sensed, so a control system would still be programmed to ignore them, but since the control matrix is different in the two cases, using a flat mirror control system will result in crosstalk between the first three badly sensed curved mirror modes and the controlled modes resulting in more error in the mirror surface. Slower mirrors and mirrors with fewer segments are closer to a flat mirror model and show less of the effect of the curvature 
than larger faster mirrors which behave more like spherical mirrors. Figure 8 shows results from a study of mirror systems with different numbers of rings and focal ratios. The left panels show a 3 ring system and the right panels show a 7 ring system. For each system three focal ratios are shown. The first row with behavior close to a flat mirror, the next row shows the transition to spherical mirror dominated behavior and the third row shows the system behaving similarly to a spherical mirror where the sensed mode shape is more like focus than the piston mode of a flat mirror.

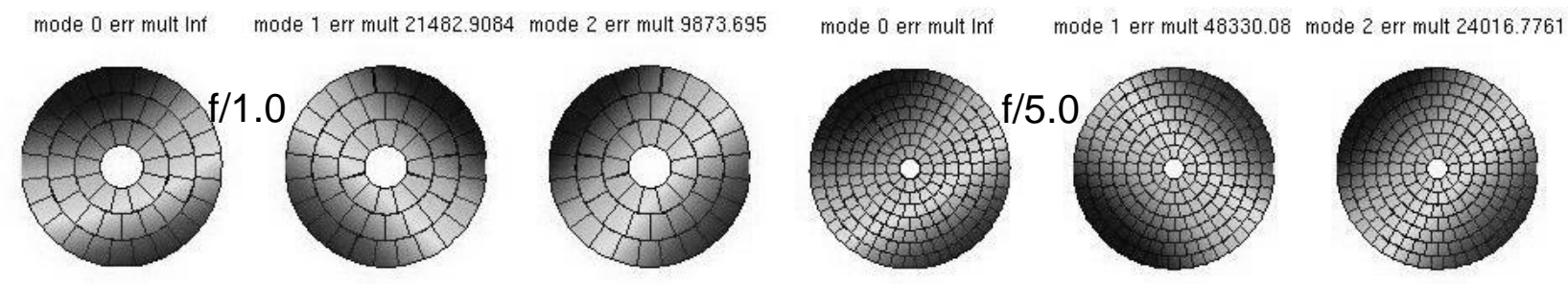

mode 0 err mult 93049.4166 mode 1 err mult 11773.8432 mode 2 err mult 5641.3703 mode 0 err mult 86175.3383 mode 1 err mult 8271.0444 mode 2 err mult 4502.479
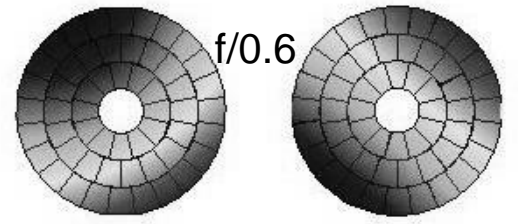

mode 0 err mult 19402.0125 mode 1 err mult 1096.3916 mode 2 err mult 1005.6059
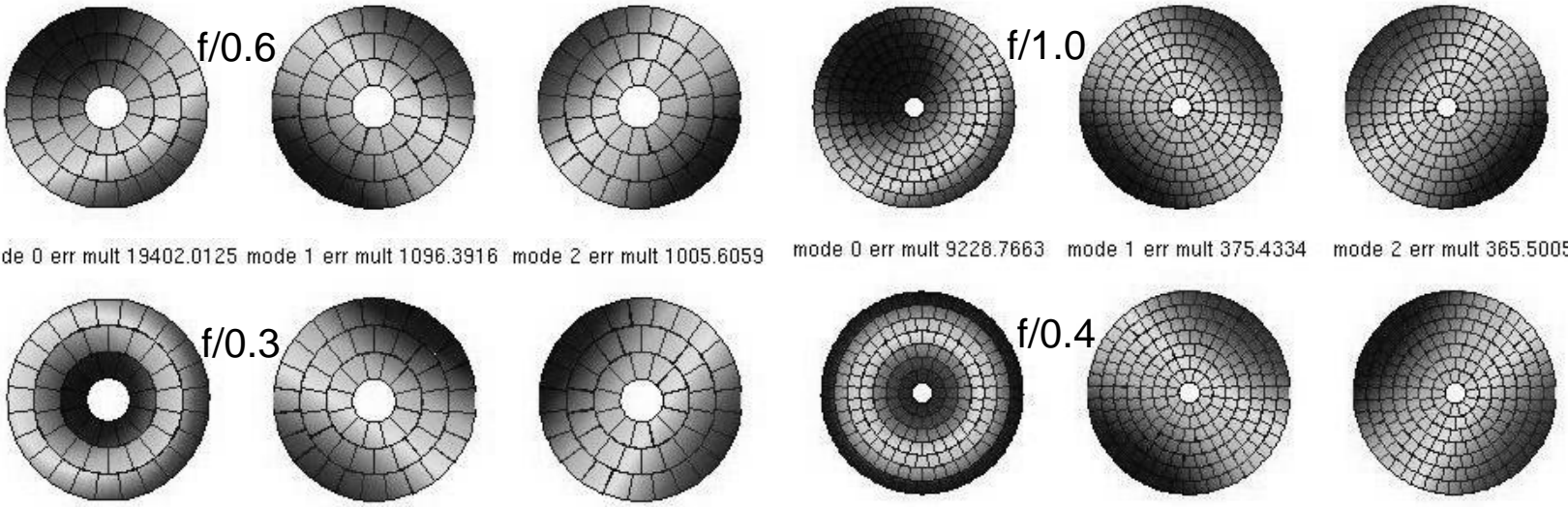

mode
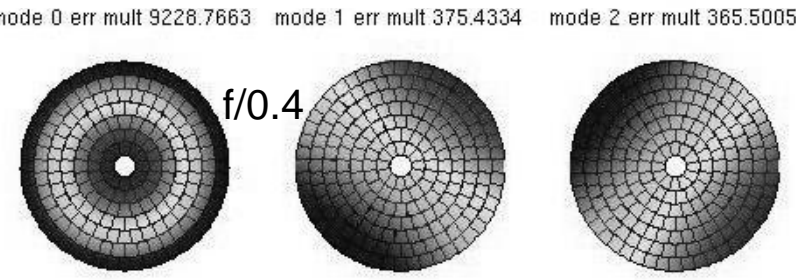

Fig. 8. Modes unsensed in flat mirrors Left -3 ring keystone Right -7 ring keystone

The behavior of the sensed modes which a control system will use are similar for flat and curved mirrors. Table 2 shows the total mirror error multipliers excluding the unsensed flat panel modes for 7 ring mirror systems with different combinations of sensors. All the combinations below the line in the table give acceptable results for a CCAT control system with the assumption of rigid panels. The rigid panel assumption comes from using the three rigid body degrees of freedom on each panel when creating the response matrix. The CCAT mirror panels will not be rigid so a panel distortion mode is also needed.

Table 2. Total error multipliers for rigid panel mirror systems

\begin{tabular}{|lrrrr|}
\hline \multicolumn{1}{|c}{ Sensors used } & \multicolumn{4}{c|}{ Error Multiplier } \\
& Flat Key & f/0.4 Key & Flat Hex & f/0.4 Hex \\
Edge 0mm offset & 867 & 748 & 477 & 371 \\
Edge 55mm offset & 16 & 16 & 6.2 & 6.5 \\
55mm edge + 4 met & 12 & 13 & 3.7 & 3.7 \\
\hline 0mm edge + dihedral & 1.7 & 1.7 & 1.2 & 1.2 \\
55mm edge + dihedral & 1.7 & 1.7 & 1.2 & 1.2 \\
0 mm edge + Shack Hartmann & 0.8 & 0.8 & 2.3 & 2.3 \\
$55 \mathrm{~mm}$ edge + Shack Hartmann & 0.8 & 0.8 & 2.3 & 2.3 \\
$55 \mathrm{~mm}$ edge + dihedral + 4 met & 1.3 & 1.2 & 0.8 & 0.8 \\
$55 \mathrm{~mm}$ edge + SH + 4 met & 0.8 & 0.8 & 0.6 & 0.6 \\
\hline
\end{tabular}




\section{THERMAL DISTORTION (CUPPING)}

Since the front surface of the mirror looks at the cold sky and the back surface looks at the warm dome, we expect thermal gradients through the mirror panels during operations. The baseline design for the CCAT panels is an aluminum honeycomb core with carbon fiber face sheets as shown in the left panel of Figure 9. A finite element model of these carbon fiber sandwich panels shows that a thermal gradient from the front to the back surface leads to cupping of the panel. For an expected gradient of $0.6^{\circ} \mathrm{C}$, the right panel in figure 9 shows that there is a $2 \mu \mathrm{m}$ distortion at the edge of the panel where sensors are placed. This means that to meet the $7 \mu \mathrm{m}$ goal for the mirror the total error multiplier for the mirror must be less than 2.5. All the sensor configurations below the line in Table 2 meet this goal for the assumption of a rigid panel. The model used to generate Table 2 was created exercising the 3 rigid body motions of the panels, so this does not reflect the case when thermal cupping is a possible motion of the panel. A new set of models were made including thermal cupping distortions in the panels as a $4^{\text {th }}$ degree of freedom.
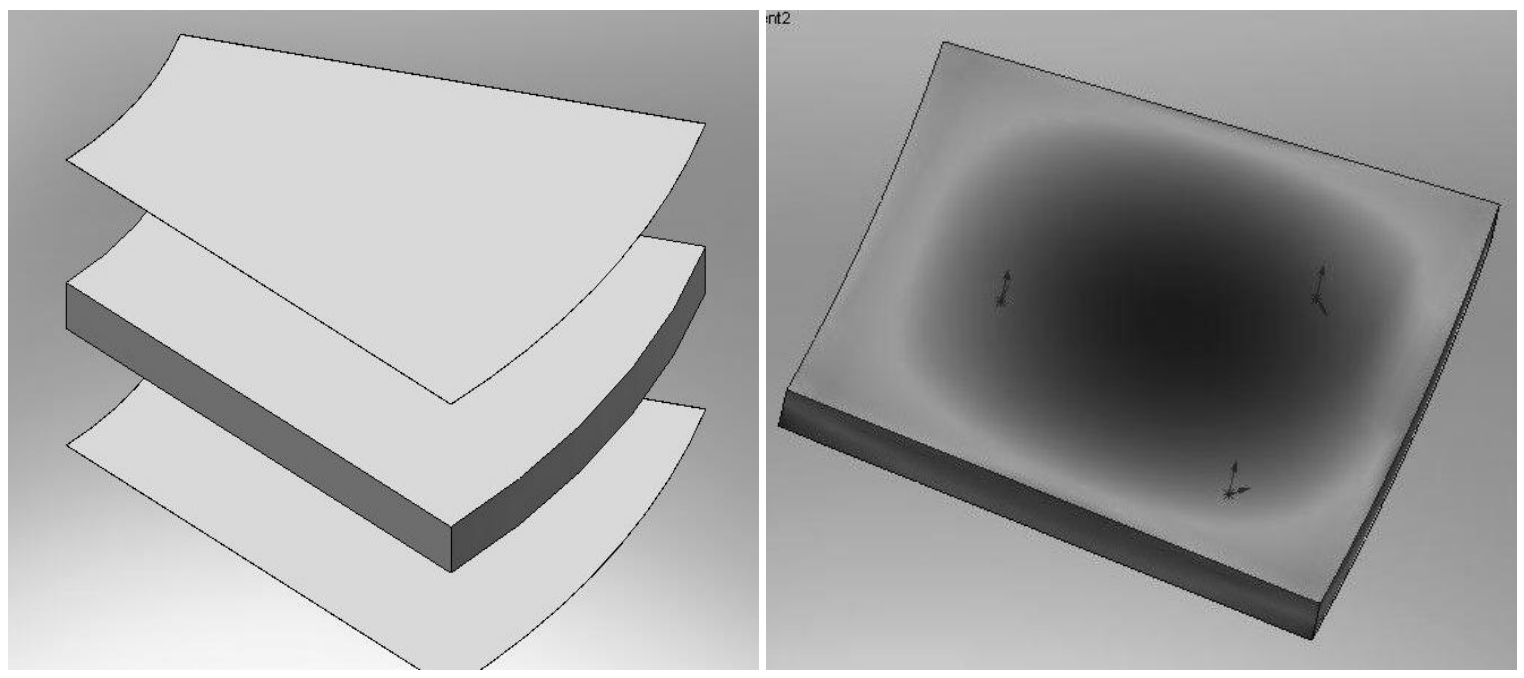

Fig.9 Left - CCAT panel exploded view Right - Thermal cupping

Table 3. Total error multipliers for 3 vs. 4 degrees of freedom

\begin{tabular}{|lrrrrr|}
\hline \multicolumn{1}{c}{ Sensors used } & \multicolumn{2}{c}{ Keystone f/0.4 } & \multicolumn{2}{c|}{ Hexagon $\mathrm{f} / 0.4$} \\
& Rigid & 4 dof & Rigid & \multicolumn{1}{c|}{4 dof } \\
0mm edge + dihedral & 1.7 & 21.77 & 1.2 & 177 \\
55mm edge + dihedral & 1.7 & 2.24 & 1.2 & 1.5 \\
0 mm edge + Shack Hartmann & 0.8 & 9.45 & 2.3 & 137.3 \\
$55 \mathrm{~mm}$ edge + Shack Hartmann & 0.8 & 1.84 & 2.3 & 1.25 \\
$55 \mathrm{~mm}$ edge + dihedral + 4 met & 1.2 & 2.1 & 0.8 & 2.33 \\
$55 \mathrm{~mm}$ edge + SH + 4 met & 0.7 & 1 & 0.6 & 1.24 \\
\hline
\end{tabular}

The results of this study, summarized in Table 3, show that increased measurement of the dihedral angle in the system now has a large effect on mirror controllability. The second and forth columns show the rigid panel model results and the third and fifth columns show the model including thermal cupping. The sensor configurations that meet the CCAT surface requirements in the presence of thermal cupping are highlighted. 


\section{CONCLUSIONS}

We find that in general hexagonal mirrors are more controllable with panel based sensors than keystone mirrors are. The effects of curvature on mirror control become more important as mirrors are designed with more panels and with

faster prescriptions. For both hexagonal and keystone mirror system we have found sensor configuration that can control the CCAT primary mirror to meet the CCAT surface requirement.

\section{ACKNOWLEDGMENTS}

This research was carried out at the Jet Propulsion Laboratory, California Institute of Technology, under a contract with the National Aeronautics and Space Administration.

\section{REFERENCES}

[1] Woody, D., MacDonald, D., Bradford, M., Chamberlin, R., Dragovan, M., Goldsmith, P., Lamb, J., Radford, S., \& Zmuidzinas, J., "Panel Options for Large Precision Radio Telescopes", 2008, in Advanced Optical and Mechanical Technologies in Telescopes and Instrumentation, ed. Atad-Ettedgui, E., \& Lemke, D., Proc. SPIE 7018

[2] Troy, M., Chanan, G., Sirko, E., and Leffert, E., "Residual misalignments of the Keck Telescope primary mirror segments: classification of modes and implications for adaptive optics," Int. Soc. Opt. Eng. 3352, 307 (1998) 